\title{
LA MATHÉMATIQUE CHEZ GEORGES PEREC ET CHEZ JORGE LUIS BORGES: UNE ÉTUDE COMPARATIVE
}

\author{
Jacques Fux \\ jacfux@gmail.com
}

Une fois de plus, je fus comme un enfant qui joue à cache-cache et qui ne sait pas ce qu'il craint ou désire le plus: rester cacher, être découvert.

Georges Perec

Il existe plusieurs livres et articles au sujet de la question des rapports entre mathématique et littérature dans les œuvres de Georges Perec, des auteurs de l'Oulipo et de Jorge Luis Borges. Les plus importants sont Unthinking thinking: Jorge Luis Borges, mathematics, and the new physics de Floyd Merrell, The unimaginable mathematics of Borges' Library of Babel de William Bloch Goldbloom, The cosmic web de N. Katherine Hayles, Borges y la matemática de Guillermo Martínez, le livre de Bernard Magné, Georges Perec, et les volumes de la Bibliothèque Oulipienne. Par contre, aucune étude ne montre les différences et les ressemblances entre la mathématique utilisée par Georges Perec et celle utilisée par Jorge Luis Borges. Dans cet article, on se propose non seulement de mettre en évidence quelques concepts et structures mathématiques différents de ceux déjà exposés dans les livres et articles cités, mais aussi d'étudier la relation entre Perec et Borges.

En mettant ainsi en relation mathématique et littérature, on soulève deux questions théoriques importantes. Celle, d'abord, de la réception etdelaproductiondestextesquiutilisentla mathématique, 
cette réflexion engageant elle-même une interrogation d'ordre méthodologique, qu'on peut formuler de la manière suivante: en quoi les outils de la littérature comparée peuvent-ils nous aider à comprendre cette connexion? On constate que l'utilisation de la mathématique et de la logique dans la production de leurs textes distinguent deux types de réceptions différents des œuvres de Perec et de Borges: celles des lecteurs qui possèdent, ou non, ce genre de connaissances. Le chercheur en littérature comparée est ainsi conduit à proposer quelques structures nouvelles pour mieux comprendre l'union de ces champs de la connaissance dans les œuvres de Borges et Perec. Ces structures nouvelles sont fondées sur la relation entre la mathématique et la littérature, c'est dire que nous ne sommes plus ni dans un domaine strictement littéraire ni dans un domaine strictement mathématique, puisqu'on cherche à comprendre l'union des deux.

Par rapport aux connaissances mathématiques on peut distinguer deux points de vue: le Platonicien et le Formaliste. Le point de vue platonicien est dominant, mais le point devue formalisteest le plus respecté philosophiquement. Les platoniciens croient que la mathématique est découverte et les formalistes croient que la mathématique est un jeu sans signifié, mais avec une infinité de possibilités. Dans le domaine littéraire, on peut considérer Borges comme un écrivain platonicien et Perec (de même que les Oulipiens) comme un écrivain formaliste. Mais la proximité mathématique entre Perec et Borges tient d'abord à la nature de leurs œuvres: il y a des références directes à la mathématique chez Perec et chez Borges, et aussi dans les témoignages qu'ils ont laissés sur leurs créations respectives. Par exemple, Borges écrit:

Revisando la biblioteca, veo con admiración que las obras que más he releído y abrumado de notas manuscritas son el Diccionario de la filosofia de Mauthner, la Historia biográfica de la filosofia de Lewes, la Historia de la guerra de 19141918 de Liddell Hart, la vida de Samuel Jonson de Boswell y la psicología de Gustav Spiller: The Mind of Man, 1902. A ese heterogéneo catálogo (que no excluye obras que tal vez son meras costumbres, como la de G.H. Lewes) preveo que los años agregarán este libro amenísimo. (BORGES, 1999, p. 85)

Ce livre auquel Borges fait référence s'intitule Mathematics and the imagination ${ }^{1}$ de Edward Kasner et James Newman. Borges écrit aussi

1. Dans les archives de l'Oulipo à la Bibliothèque de l'Arsenal, on trouve un exemplaire du même livre. Il y a aussi une traduction qui s'appelle Les mathématiques et l'imagination, 
dans le même texte: "Sus cuatrocientas páginas registran con claridad los inmediatosy accesibles encantos de las matemáticas, los que hasta un mero hombre de letras puede entender, o imaginar que entiende" (BORGES, 1957, p. 165). Les concepts présents dans ce livre sont vraiment utilisés par Borges dans ses œuvres. Perec écrit également sur les mathématiques et les contraintes:

Je me souviens de la théorie mathématique de la transitivité. Je me souviens que tous les nombres dont les chiffres donnent un total de neuf sont divisibles par neuf (parfois, je passais des après-midi à le vérifier). (PEREC, 1978b, p. 10) La poésie, c'était compter sur ses doigts jusqu'à douze. La chose ne se faisant plus aussi innocemment depuis pas mal de temps [...], j'ai choisi d'appeler "poésie" des textes engendrés par des contraintes difficiles². (MAGNÉ, 1999, p. 65)

Il faut remarquer que Borges et Perec avaient des références mathématiques. Borges connaissait, même avant sa lecture du livre Mathematics and the imagination, quelques concepts logiques et mathématiques (1933): "La expansión del universo de Sir Arthur Stanley Eddington"; 1934: "La cuarta dimensión" et "La doctrina de los ciclos; 1936: "Historia de la eternidad"; 1938: "Men of mathematics, de E.T Bell" et "Un resumen de las doctrinas de Einstein" (CAMURATI, 2005, p. 136) et Perec a beaucoup travaillé avec les mathématiciens de l'Oulipo. "Perec était fermement persuadé que son écriture restait le fruit d'une technique et non pas un don du génie. Il rejetait catégoriquement l'image, héritée du romantisme, de l'écrivain laissant parler sa muse dans une mansarde éclairée par une chandelle" (BELLOS, 1994, p. 380). La technique, donc, chez Perec et Borges, est parfois de nature mathématique.

Dans Prólogos, Borges a écrit:

la imaginación y las matemáticas no se contraponen; se complementan como la cerradura y la llave. Como la música, las matemáticas pueden prescindir del Universo, cuyo ámbito comprenden y cuyas ocultas leyes exploran. (BORGES, 1985b, p. 36)

Borges a mis en œuvre le concept d'infini dans le texte "El Aleph" et Perec a écrit un livre sans la lettre $e$, La disparition $^{3}$. On peut dire que

Trad. de Francine Beris et François Le Lionnais. Éd. Payot, coll. Bibliothèque scientifique. Paris, 1970.

2. Bien sûr que les contraintes difficiles auxquelles fait allusion Perec ne sont pas nécessairement toutes mathématiques.

3. Si nous considérons la mathématique comme un système axiomatique, nous pouvons considérer aussi le lipogramme comme un axiome initial. 
Borges a utilisé un concept mathématique, alors que Perec a utilisé une contrainte ou un axiome mathématique. Les différences principales résident dans la présentation des textes; Perec et les Oulipiens écrivent avec des contraintes et cela apparaît dans la structure du texte (par exemple: palindromes, lipogrammes, baobab, etc.); Borges, en se rapportant à un concept mathématique, comme l'énumération d'un ensemble infini, écrit sa fiction sur "Le livre de sable", par exemple, et travaille sur l'absence de quelques pages dans ce livre.

Dans cet article, on se propose non seulement de mettre en évidence quelques concepts et structures mathématiques différents de ceux déjà exposés dans les livres et articles cités, mais aussi d'étudier la relation entre Perec et Borges.

\section{L'EUVRE DE PEREC ET SES RAPPORTS AVEC CELLE DE BORGES}

L'Ouvroir de Littérature Potentielle (Oulipo) est un groupe composé d'écrivains et de mathématiciens qui travaille systématiquement avec la mathématique dans la littérature. Ils ont eu l'idée "injecter des notions mathématiques inédites dans la création romanesque ou poétique" (OULIPO, 1981, p. 22). La démarche de l'Oulipo, selon les Oulipiens, se divise en deux catégories: l'anoulipisme et le synthoulipisme. Nous pouvons rapprocher Borges de la catégorie de l'anoulipisme (analytique):

On peut distinguer dans les recherches qu'entend entreprendre l'Ouvroir, deux tendances principales tournées respectivement vers l'Analyse et la Synthèse. La tendance analytique travaille sur les œuvres du passé pour y rechercher des possibilités qui dépassent souvent ce que les auteurs avaient soupçonné. C'est, par exemple, le cas du centon qui pourrait, me semble-t-il, être revigoré par quelques considérations tirées de la théorie des chaînes de Markov. (OULIPO, 1973, p. 17)

La catégorie du synthoulipisme classe Perec comme un écrivain qui utilise vraiment la mathématique dans ses œuvres et met en évidence la différence entre la mathématique de Borges et celle de Perec:

La tendance synthétique est plus ambitieuse; elle constitue la vocation essentielle de l'Oulipo. Il s'agit d'ouvrir de nouvelles voies inconnues de nos prédécesseurs. C'est, par exemple, le cas de Cent Mille Milliards de Poèmes ou des haï-kaï booléens. Les mathématiques - plus particulièrement les structures abstraites des mathématiques contemporaines - nous proposent mille directions d'explorations, tant à partir de l'Algèbre (recours à de nouvelles lois de composition) que de la Topologie (considérations de voisinage, d'ouverture 
ou de fermeture de textes). Nous songeons aussi à des poèmes anaglyphiques, à de textes transformables par projection, etc. D'autres raids peuvent êtres imaginés, notamment dans le domaine des vocabulaires particuliers (corbeaux, renards, marsouins; langage Algol des ordinateurs électroniques, etc.) (OULIPO, 1973, p. 17-18).

Selon François Le Lionnais: "En résumé, l'anoulipisme est voué à la découverte, le synthoulipisme à l'invention. De l'un à l'autre existent maints subtils passages" (OULIPO, 1973, p. 18). Borges était un découvreur et un inventeur, on peut le qualifier d'écrivain potentiel; quant à Perec, il a relu et médité l'œuvre de Borges, et a également inventé de nouvelles contraintes pour obtenir des matrices littéraires potentielles.

L'article du mathématicien Claude Berge définit les vocations de l'Oulipo (analytique, synthétique) et aussi la troisième qui "consiste en la transposition dans le domaine des mots des concepts existant dans les différentes branches des mathématiques: géométrie (poèmes tangents entre eux de Le Lionnais), algèbre de Boole (intersection de trois romans, de J. Duchateau), algèbre matricielle (multiplication de textes de Queneau), etc." (OULIPO, 1973, p. 50). La relation (mathématique) qui existe entre Perec et Berge est, d'ailleurs, bien connue: "Claude Berge a collaboré avec Georges Perec à qui il a proposé l'usage du bicarré latin d'ordre 10 pour répartir les attributs dans les différentes pièces de l'immeuble décrit dans La vie mode d'emploi" (oulipo.net). On peut y ajouter aussi l'analyse mathématique (les concepts de l'infini chez Borges). Les mathématiques peuvent donc être regardées comme un renouvellement de l'invention littéraire.

Queneau, dans son article "La place des mathématiques dans la classification des sciences", présente le développement des travaux oulipiens comme similaire à celui de la recherche mathématique: "l'analyse des cryptogrammes, des acrostiches, etc., devrait aussi, à mon avis, enrichir la nouvelle rhétorique de la même manière que certains types de casse-tête ont conduit à l'introduction de la théorie des probabilités, de la topologie et de la théorie des jeux" (QUENEAU, 1998, p. 393).

Dans son article "Georges Perec et les mathématiques", Bernard Magné montre que Georges Perec n’aimait pas beaucoup la mathématique et que la mathématique de Georges Perec n'a rien à voir avec celle de Raymond Queneau, ou de Jacques Roubaud. Perec a surtout utilisé trois règles mathématiques et, en fait, comme dans toute son œuvre, s'est livré à une tentative d'épuisement de leurs différentes possibilités littéraires. En utilisant un concept mathématique, on peut appeler cet épuisement méthode d'exhaustion (c'est une méthode de calcul). Dans sa préface aux Romans et récits de Georges Perec, Bernard Magné écrit: 
Les contraintes de La Vie mode d'emploi sont remarquables non seulement par leur nombre mais aussi par leur nouveauté dans le champ de la littérature: des trois processus formels aux énoncés alléchants, un seul a une ascendance rhétorique: la pseudo-quenine, avatar, grâce à Raymond Queneau puis Jacques Roubaud, d'une forme poétique médiévale, la sextine du troubadour Arnaut Daniel. La polygraphie du cavalier vient, comme son nom l'indique, des échecs et le bi-carré latin de la combinatoire mathématique. Perec n'invente pas, à proprement parler, les contraintes principales de La Vie mode d'emploi, mais il va les puiser hors de l'héritage littéraire traditionnel (MAGNÉ, 2002, p. 20).

Quant aux œuvres de Borges, elles travaillent sur la littérature, sur les réseaux littéraires, sur les écrivains et l'héritage en utilisant plusieurs concepts soit philosophiques, soit fictionnels, ou bien mathématiques. Perec travaille aussi directement avec les citations, les références littéraires et, pour cela, il applique quelquefois des procédures mathématiques.

Selon la définition du terme, l'exhaustion est l'action d'épuiser tous les cas possibles dans une question donnée. En mathématique, la méthode d'exhaustion est la manière de prouver que deux grandeurs sont égales, en montrant que la différence en est plus petite que toute quantité donnée. La méthode d'exhaustion repose sur le principe souvent appelé axiome d'Archimède: en soustrayant de la plus grande de deux grandeurs données plus de sa moitié, et du reste plus de sa moitié, et ainsi de suite, on obtiendra une grandeur moindre que la plus petite. Perec a utilisé ces concepts en particulier dans Espèces d'espaces, Théâtre I, Tentative d'épuisement d'un lieu parisien, Cantatrix Sopranica L., et autres écrits scientifiques, et dans La vie mode d'emploi.

Lerapprochementdeladémarcheperecquienneetdela mathématique est bien sûr à nuancer dans la mesure où Perec était tout à fait ignorant des mathématiques, et il est donc difficile de dire qu'il a véritablement "mis en œuvre" des concepts ou des résultats mathématiques. En revanche, Perec a souvent utilisé la mathématique en tant que membre de l'Oulipo, et passionné de jeux et de contraintes. Par exemple, on l'a déjà rappelé, la structure du bicarré latin utilisée dans La vie mode d'emploi lui a été donnée par Claude Berge, mathématicien membre de l'Oulipo. Claude Berge raconte: "En 1967, au cours d'une réunion de l'Oulipo, j'eus l'occasion d'en parler à Georges Perec qui réalisa alors sous le titre 'Carrés latins' une première ébauche de ce qui devait devenir La vie mode d'emploi” (MAGNÉ, 2002, p. 643).

De manière générale, l'invention mathématique dans l'œuvre de Perec résulte toujours d'une collaboration. Pour La disparition, Perec a demandé à Jacques Roubaud d'écrire un texte mathématique lipogrammatique et, 
dans La vie mode d'emploi, Perec a recopié (fautivement) un extrait de la thèse de mathématiques de Jacques Roubaud.

\begin{abstract}
Aux Maths:
La notion-là, qui la conquit, qui la trouva, qui la fournit? Gauss ou Galois? L'on n'a jamais su. Aujourd'hui, tout un chacun connaît ça. Pourtant, on dit qu'au fin fond du noir, avant sa mort, dans la nuit, Galois grava sur son pad un long chaînon à sa façon. Voici:

$a a^{-1}=b b^{-1}=c c^{-1}=d d^{-1}=f f^{1}=g g^{-1}=h h^{-1}=i i^{-1}=j j^{-1}=k k^{-1}=l l^{-1}=m m^{-1}=n n^{-1}=o 0^{-1}=p p^{-1}=r r^{-1}=$ $s s^{-1}=t t^{-1}=u u^{-1}=v v^{-1}=w w^{-1}=x x^{-1}=y y^{-1}=z z^{-1}$ (PEREC, 2002, p. 351).

Si $f \in \operatorname{Hom}(v, \mu)$ (resp. $G$ Hom $(\xi, v))$ est un morphisme homogène dont le degré est la matrice $\alpha$ (resp. $\beta$ ), $f$ o $g$ est homogène et son degré est matrice produit $\alpha \beta$ (PEREC, 2002, p. 662).
\end{abstract}

Jacques Roubaud a été le premier membre de l'Oulipo vraiment mathématicien et écrivain; "non seulement il avait été à la fois poète et mathématicien bien avant d'avoir entendu parler de l'Oulipo, mais il était même plus précisément un vrai poète-mathématicien, dont les travaux se situaient à l'intersection des jeux, des langages formels et du langage tout court" (BELLOS, 1994, p. 380).

Les mathématiciens amateurs qui apparaissent dans La vie mode emploi ${ }^{4}$ sont plutôt obsessionnels, comme tous les autres personnages. Perec lui-même, comme on sait, avait l'ambition avouée de "remplir un tiroir de la Bibliothèque nationale, d'utiliser tous les mots de la langue française, d'écrire tout ce qu'il est possible à un homme d'aujourd'hui d'écrire" (MAGNÉ, 2002, p. 12).

Ilya en mathématique une forme de raisonnement nommée induction mathématique ou raisonnement par récurrence, visant à démontrer une propriété portant sur tous les entiers naturels. Le raisonnement par récurrence consiste à démontrer les points suivants:

- Une propriété est satisfaite par l'entier o ou pour le premier terme d'une série (progression);

- $\quad$ Si cette propriété est satisfaite par un certain nombre entier naturel $n$, alors elle doit être satisfaite par son successeur, c'est-àdire le nombre entier $n+1$.

Une fois cela établi, on en déduit cette propriété pour tous les nombres entiers naturels.

4. Il y en a quelques-uns, par exemple: Mortimer Smautf, Carel Van Loorens, Abel Speiss. 
L'aspect encyclopédique de l'œuvre de Georges Perec peut être rapproché de l'aspect inductif et épuisant (ancienne démonstration utilisée) de la mathématique. Perec voulait utiliser tous les mots de la langue, toutes les possibilités du langage, la potentialité mathématique et son processus de création (formaliste). Les formalistes croient que la mathématique n'existe pas antérieurement, que nous pouvons construire des règles logiques et utiliser ensuite toutes leurs possibilités. Ce que la logique est à la mathématique, les mots le sont à Perec. Le procès de l'induction mathématique vise à trouver une solution pour tous les nombres naturels et les œuvres de Perec visent à épuiser toutes les possibilités langagières. Cependant, ni Perec ni les mathématiciens ne parviennent à leurs fins.

Perec avait également une obsession pour les nombres, il était "un manipulateur de chiffres ou de nombres, comme il est manipulateur de lettres ou de mots" (MAGNÉ, 2002, p. 15). Dans ses œuvres, on rencontre un certain nombre de références aux chiffres. Dans Je me souviens, il écrit: "Je me souviens que tous les nombres dont les chiffres donnent un total de neuf sont divisibles par neuf (parfois, je passais des après-midi à le vérifier)" (PEREC, 1978b, p. 285). Dans 53 jours, il écrit sur les neuf manières dont le nombre 53 peut faire partie d'une suite de Fibonacci. Dans le vers 43 du Compendium de La vie mode d'emploi, il y a une référence à la conjecture de Goldbach: "Le Hollandais disant que tout nombre est somme de K premiers. Non seulement on retrouve ici une allusion aux nombres premiers, dont on sait l'importance dans la symbolique perecquienne, mais encore l'intervention du $\mathrm{K}$ dans ce contexte semble bien être une invention: ce vers 43 se présente en effet comme un écho du chapitre LXXVIII où il est question d'une 'note sur le problème de Goldbach, proposant que tout nombre $\mathrm{n}$ soit la somme de $\mathrm{K}$ nombres premiers"” (MAGNÉ, 1999, p. 65). Or, le souvenir mathématique inclus dans Je me souviens est incomplet ${ }^{5}$, d'un point de vue mathématique. En fait, un nombre est divisible par 9 si la somme de ses chiffres est divisible par 9. Par exemple, 99 est divisible par 9 parce que la somme 9+9 $=18$ et 18 est divisible par 9 (mais 9+9 ne donne pas un total de 9, comme Perec l'écrit).

Comme on l'a déjà dit, les formalistes croient que la mathématique est un jeu sérieux, difficile et qui met en œuvre des aspects logiques, des

5. La remarque faite par Perec n'est pas du tout fausse mais incomplète. Il est parfaitement exact, comme le dit Perec, que tous les nombres dont les chiffres donnent un total de 9 sont divisibles par 9. Ce qui n'est pas vrai, c'est la réciproque (mais il ne le dit pas). 
raisonnements. Perec, en utilisant cette partie de la mathématique, a élargi les possibilités de lecture de ses œuvres.

Chez Perec, le lipogramme, le palindrome et les contraintes issues du jeu d'échecs peuvent être présentés comme de nature mathématique; selon Christelle Reggiani "on est alors face à une conception instrumentale du rapport entre mathématiques et invention littéraire: les mathématiques seraient retenues par la démarche oulipienne en raison de leur caractère formel, évidemment intéressant pour une littérature qui s'écrit essentiellement autour de structures" (REGIANNI, 1998, p. 58). Plus précisément, la démarche oulipienne suit le modèle proposé par Bourbaki: "l'entreprise oulipienne se pose autrement dit comme une refondation axiomatique de la littérature, sur le modèle de celle opérée, pour les mathématiques, par Bourbaki" (REGIANNI, 1998, p. 59).

On a montré quelques exemples de l'utilisation de la mathématique par Georges Perec: on voit qu'il a utilisé la mathématique non seulement en y faisant des références, mais aussi comme un outil pour composer vraiment des textes. C'est, à mon avis, la chose la plus importante qui distingue les travaux de Borges et ceux de Perec. Perec a donc utilisé la mathématique alors qu'il n'en avait pas de connaissance approfondie, et c'est aussi le cas de Borges.

\section{BORGES ET LA MATHEMATIQUE. RELATIONS AVEC L'EUVRE DE PEREC}

Jorge Luis Borges a utilisé différents concepts pour créer ses fictions. Les concepts les plus importants pour faire le lien entre la mathématique de Borges et son écriture sont la Kabbale, les paradoxes grecs et l'analyse mathématique.

Comme Perec, Borges connaissait peu la mathématique et a cherché à épuiser ces concepts, selon Hayles:

Borges attempts to increase rather than use up the available permutations. Instead of hundreds of pages he writes five or six, characteristically including at least an open-ended catalogue capable of indefinite expansion. For Borges stasis is impossible because art is not an object to be framed, but a continuing process whose permutations are inexhaustible. Borges's work is so repetitive: the same themes, ideas, and paradoxes keep recurring (HAYLES, 1984, p. 138).

Le paradoxe, du grec paradoxos ( $\pi \alpha \rho \alpha ́ \delta$ ołoc), signifie "contraire à l'opinion commune, bizarre, extraordinaire”; c'est une proposition 
qui contient ou semble contenir une contradiction logique, ou un raisonnement qui, bien que sans faille apparente, aboutit à une absurdité, ou encore une situation qui contredit l'intuition commune (ROSSET, 2004, p. 110). Selon Hayles:

\begin{abstract}
What fascinates Borges is the prospect of a set that contains itself, a whole that both contains and is contained by the part. Such paradoxes are implicit in many representations of field models, because the representation is at once the whole, in the sense that it images the field, and the part, in the sense that it is contained within the whole it figures. This paradox, central to Borges's fictions, is explored through the infinite sets and transfinite numbers of Cantor set theory. Borges's assumption is that the Newtonian universe must crumble when confronted with antinomies to which this theory gave rise. But he does not want a new reality to come into being either. Rather, he juxtaposes the new "loss of certainty" with old certainties to render everything uncertain (HAYLES, 1984, p. 27).
\end{abstract}

Borges connaissait quelques paradoxes de la philosophie et de la mathématique. L'utilisation de ces concepts se retrouve dans les textes suivants: "El idioma analítico de John Wilkins", "Examen de la obra de Herbert Quain”, "La biblioteca de Babel”, "La lotería de Babilonia”, "La esfera de Pascal", "Avatares de la tortuga”, "La doctrina de los ciclos”, "Argumentum Ornithologicum", "El disco" et "La muerte y la brújula”.

À l'origine, les paradoxes semblent inoffensifs et amusants, mais leur résolution (soit philosophique soit mathématique) est très compliquée, et très importante pour le développement des connaissances.

Le paradoxe de Bertrand Russell peut être formulé ainsi: l'ensemble des ensembles n'appartenant pas à eux-mêmes appartient-il à luimême? Si l'on répond oui, alors, comme par définition les membres de cet ensemble n'appartiennent pas à eux-mêmes, il n'appartient pas à lui-même: contradiction. Mais si l'on répond non, alors il a la propriété requise pour appartenir à lui-même: contradiction encore. On a donc une contradiction dans les deux cas, ce qui rend l'existence d'un tel ensemble paradoxale. Réécrit plus formellement, si l'on pose:

$y=\{x \mid x \square x\}$ on a immédiatement que $y \in y \square y \square y$, donc chacune des deux possibilités, $y \in y$ et $y \square y$, mène à une contradiction (Russell).

Borges écrit "La perpetua carrera de Aquiles y la tortuga”:

El límite de la suma de esta infinita progresión geométrica es doce (más exactamente, once y un quinto; más exactamente, once con tres veinticincoavos), pero no es alcanzado nunca. Es decir, el trayecto del héroe 
será infinito y éste correrá para siempre, pero su derrotero se extenuará antes de doce metros, y su eternidad no verá la terminación de doce segundos. Esa disolución metódica, esa ilimitada caída en precipicios cada vez más minúsculos, no es realmente hostil al problema: es imaginárselo bien. No olvidemos tampoco de atestiguar que los corredores decrecen, no sólo por la disminución visual de la perspectiva, sino por la, disminución admirable a que los obliga la ocupación de sitios microscópicos. Realicemos también que esos precipicios eslabonados corrompen el espacio y con mayor vértigo el tiempo vivo, en su doble desesperada persecución de la inmovilidad y del éxtasis (BORGES, 2002a, p. 244).

En 1900, David Hilberta proposéla résolution de vingt-trois problèmes (les fameux problèmes de Hilbert), qui étaient ouverts (certains ne sont toujours pas résolus aujourd'hui), pour montrer qu'en mathématique il n'y a pas d'ignorabimus. En 1931, Gödel a prouvé que le rêve de Hilbert était impossible en démontrant qu'en mathématique il y a des énoncés qui sont vrais bien qu'il soit impossible d'en démontrer la véracité.

Borges a énoncé lui aussi qu'il y a des choses qu'on peut concevoir sans pouvoir les résoudre. Dans "Argumentum Ornithologicum”, il présente ainsi ses concepts mathématiques et logiques:

Cierro los ojos y veo una bandada de pájaros. La visión dura un segundo o acaso menos; no sé cuántos pájaros vi. ¿Era definido o indefinido su número? El problema involucra el de la existencia de Dios. Si Dios existe, el número es definido, porque Dios sabe cuántos pájaros vi. Si Dios no existe, el número es indefinido, porque nadie pudo llevar la cuenta. En tal caso, vi menos de diez pájaros (digamos) y más de uno, pero no vi nueve, ocho, siete, seis, cinco, cuatro, tres o dos. Vi un número entre diez y uno, que no es nueve, ocho, siete, seis, cinco, etcétera. Ese número entero es inconcebible; ergo, Dios existe (BORGES, 2002b, p.165).

\section{Dans "El libro de arena", Borges présente le concept qu'il a l'intention d'utiliser dans ce texte:}

La línea consta de un número infinito de puntos; el plano, de un número infinito de líneas; el volumen, de un número infinito de planos; el hipervolumen, de un número infinito de volúmenes... No, decididamente no es éste, more geométrico, el mejor modo de iniciar mi relato. Afirmar que es verídico es ahora una convención de todo relato fantástico; el mío, sin embargo, es verídico (BORGES, 2002c, p. 52).

\section{Il expose ensuite le problème du livre de sable:}

Examiné con una lupa el gastado lomo y las tapas, y rechacé la posibilidad de algún artificio. Comprobé que las pequeñas ilustraciones distaban dos mil páginas una de otra. Las fui anotando en una libreta alfabética, que no tardé en 
llenar. Nunca se repitieron. De noche, en los escasos intervalos que me concedía el insomnio, soñaba con el libro. Declinaba el verano, y comprendí que el libro era monstruoso. De nada me sirvió considerar que no menos monstruoso era yo, que lo percibía con ojos y lo palpaba con diez dedos con uñas. Sentí que era un objeto de pesadilla, una cosa obscena que infamaba y corrompía la realidad. Pensé en el fuego, pero temí que la combustión de un libro infinito fuera parejamente infinita y sofocara de humo al planeta (BORGES, 2002C, p. 54).

Au sujet de l'importance de l'utilisation des concepts mathématiques et logiques chez Borges, Hayles écrit:

Borges is the first writer in this study who consistently wants to exploit rather than suppress these inconsistencies, because he hopes to use them to reveal the essential fictionality of the model. His intent is thus subversive. His strategy is seduction, for he progresses to this revelation by several seemingly innocuous steps. The first step in his strategy is to transform a continuity into a succession of points, and to suggest that these points form a sequence; there follows the insinuation that the sequence progresses beyond the expected terminus to stretch into infinity; then the sequence is folded back on itself, so that closure becomes impossible because of the endless, paradoxical circling of a selfreferential system. This complex strategy (which may not appear in its entirety in any given story) has the effect of dissolving the relation of the story to reality, so that the story becomes an autonomous object existing independently of any reality. The final step is to suggest that our world, like the fiction, is a selfcontained entity whose connection with reality is problematic or nonexistent (HAYLES, 1984, p. 143).

On constate à nouveau, de ce point de vue, les différences et les ressemblances entre Borges et Perec.

\section{BORGES ET PEREC}

On peut montrer quelques différences et ressemblances entre Perec et Borges selon des points mathématiques distincts. Par rapport à la philosophie, Borges peutêtre considérécomme platonicien puisqu'il utilise la mathématique comme outil de découvertes; sa potentialité est dans la découverte. Par contre, Georges Perec exploite des outils mathématiques pour écrire et pour en jouer dans l'écriture. Par rapport à l'utilisation de la mathématique, Borges utilise des concepts mathématiques, surtout des concepts abstraits pour augmenter ses possibilités d'écriture et de lecture. Perec utilise la mathématique dans la structure de ses écrits en ayant recours à des modèles simples (lipogramme, palindrome) ou à des modèles plus inventifs (bicarré, polygraphie du cavalier). L'écriture de Borges est fictionnelle, surtout quand il écrit ses textes de l'Aleph et 
de Ficcionnes. En revanche, Perec essaie de mettre en œuvre toutes les possibilités, tous les mots, toutes les variations en utilisant la combinatoire. Borges a acquis ses connaissances mathématiques en lisant Mathematics and the imagination (entre autres livres) alors que Perec s'est familiarisé avec la mathématique en étant membre de l'Oulipo. En ce qui concerne les problèmes mathématiques, on peut dire que la mathématique de Borges nous rend sensibles aux problèmes de l'énumération en utilisant seulement l'ensemble des nombres naturels (comme dans "El Aleph" et "El libro de arena"). Quant à Perec, il travaille avec les problèmes de la classification des livres et de toutes sortes d'objets (Penser/Classer).

Chez Borges comme chez Perec, on trouve aussi une référence aux mathématiques appliquées, et plus précisément à l'informatique. Perec a écrit Die Maschine et L'art et la manière d'aborder son chef de service pour lui demander une augmentation; quant à Borges, il a écrit “El laberinto", où il utilise le concept de récursivité:

Este es el laberinto de Creta. Este es el laberinto de Creta cuyo centro fue el Minotauro. Este es el laberinto de Creta cuyo laberinto fue el Minotauro que Dante imaginó como un toro con cabeza de hombre y en cuya red de piedra se perdieron tantas generaciones como María Kodama y yo nos perdimos. Este es el laberinto de Creta cuyo laberinto fue el Minotauro que Dante imaginó como un toro con cabeza de hombre y en cuya red de piedra se perdieron tantas generaciones como María Kodama y yo nos perdimos en aquella mañana y seguimos perdidos en el tiempo, ese otro laberinto (BORGES, 1985a, p. 36).

Dans cet extrait, Borges construit lentement une récursivité, ou une récurrence. À chaque ligne, il ajoute de nouvelles informations sur le labyrinthe de Crète. À la fin, apparaît un autre labyrinthe pour recommencer une nouvelle itération. Selon l'article "La Littérature récurrente", "le mot récurrence évoque un mouvement d'éternel retour, de répétition non bornée. Nous voudrions sous le titre 'littérature récurrente' élargir le thème en nous inspirant de la connotation mathématique qui se manifeste dans des notions comme 'démonstration par récurrence,' 'fonctions récursives', etc. Nous classerons donc sous la rubrique 'littérature récurrente' tout texte contenant, explicitement ou implicitement, des règles d'engendrement qui invitent le lecteur (ou le diseur ou le chanteur), à poursuivre la production du texte à l'infini (ou jusqu'à épuisement de l'intérêt ou de l'attention)" (OULIPO, 1981, p. 82). C'est exactement ce qu'a fait Borges avec ses labyrinthes.

Un algorithme "est une suite ordonnée d'instructions écrites dans un langage informatique et devant être traitées par un ordinateur; il se présente généralement comme un organigramme pour la compréhension 
humaine. Pour L'augmentation, la commande passée à Perec consistait à rédiger sous forme de texte l'organigramme représentant les étapes qu'un employé subalterne doit franchir pour obtenir une augmentation de salaire" (BELLOS, 1994, p. 431). Perec a donc construit un texte de 57 pages où il applique la structure algorithmique pour penser les différentes manières d'aborder son chef pour lui demander une augmentation.

Dans Die Maschine, Perec, au-delà du vieux problème cartésien est-ce que j'existe? comment est-ce que je le sais? - travaille aussi avec la langue, conçue comme un système de signes, et l'axiomatisation y ressemble au système logique de la machine de Turing (TURING, 1936, p. $42)$.

Borges a également écrit un texte qui s’intitule "El jardín de senderos que se bifurcan”, où il applique le concept d'organigramme algorithmique. Dans ce texte, Borges écrit:

La explicación es obvia: El jardín de senderos que se bifurcan es una imagen incompleta, pero no falsa, del universo tal como lo concebía Ts'ui Pên. A diferencia de Newton y de Schopenhauer, su antepasado no creía en un tiempo uniforme, absoluto. Creía en infinitas series de tiempos, en una red creciente y vertiginosa de tiempos divergentes, convergentes y paralelos. Esa trama de tiempos que se aproximan, se bifurcan, se cortan o que secularmente se ignoran, abarca todas las posibilidades. No existimos en la mayoría de esos tiempos; en algunos existe usted y no yo; en otros, yo, no usted; en otros, los dos. En éste, que un favorable azar me depara, usted ha llegado a mi casa; en otro, usted, al atravezar el jardín, me ha encontrado muerto; en otro, yo digo estas mismas palabras, pero soy un error, un fantasma. - En todos —articulé no sin un temblor- yo agradezco y venero su recreación del jardín de Ts'ui Pên. -No en todos -murmuró con una sonrisa-. El tiempo se bifurca perpetuamente hacia innumerables futuros. En uno de ellos soy su enemigo (BORGES, 2002a, p. 576).

L'importance de la logique, et la mise en avant du raisonnement, constitue ainsi un lien manifeste entre les deux écrivains.

\section{LA PRODUCTION ET LA RECEPTION DES TEXTES UTILISANT LA LOGIQUE ET LA MATHEMATIQUE}

Par rapport à la production et à la réception du texte, Perec écrit:

Une fois de plus, les pièges de l'écriture se mirent en place. Une fois de plus, je fus comme un enfant qui joue à cache-cache et qui ne sait pas ce qu'il craint ou désire le plus: rester caché, être découvert (PEREC, 1975, p. 18). 
De fait, Perec, à travers la publication d'un certain nombre d'entretiens, a révélé une part de ses contraintes et de leurs énigmes. À l'inverse, Borges ne s'est jamais préoccupé de révéler les outils utilisés dans ses œuvres, mais il se présente aussi comme un lecteur de lui-même, se dédoublant en auteur et lecteur. Comme l'écrit Jean-Yves Pouilloux, la présence des paradoxes chez Borges représente une critique de la position de l'auteur aussi bien que de celle du lecteur:

On retrouve la même ambigüité que dans hacedor, "artisan" et "faiseur". Ce qui laisse entendre que sont annoncées sous ce titre bref des histoires en forme, inventées à plaisir mais aussi, peut-être, des feintes, des trompe-l'œil, des supercheries. En sorte que, par cette double ambigüité, le contrat de lecture se trouve malaisé à définir: ni la figure de l'auteur ni le titre n'offrent les repères conventionnels propres à rassurer le lecteur par les limites qu'ils ont habituellement pour fonction de poser. Privée de ces repères, la lecture se voit d'avance condamnée à flotter dans un monde incertain et instable où les certitudes conventionnelles sont ébranlées, sinon invalidées, et où règne la perplexité: un peu comme devant le fameux aphorisme attribué à Épiménide le Crétois déclarant que "tous les Crétois sont menteurs", exemple canonique de raisonnement circulaire puisque si Épiménide dit vrai en disant que les Crétois sont menteurs, alors lui ne ment pas, bien que Crétois, et donc il ment puisque tous les Crétois mentent. C'est là, sans doute, dans la tradition occidentale, l'un des plus anciens paradoxes logiques, et il a été rangé au Moyen Âge dans les insolubilia, questions impossibles à résoudre. Mais ici il ne s'agit pas seulement d'un problème logique; en choisissant son titre, Borges suggère que ce paradoxe concerne directement la fiction en tant que telle, dans sa relation à la vérité, c'est-à-dire dans les positions respectives de l'auteur, du texte et du lecteur; et il le concerne en effet nécessairement dès l'instant où ces trois entités se voient refuser une place fixe (POUILLOUX, 1992, p. 17-18).

La réception d'œuvres qui utilisent la logique et la mathématique, comme c'est le cas chez les auteurs de l'Oulipo, et notamment Perec, et Borges est particulièrement intéressante. On peut, bien sûr, lire les sonnets "de base" des Cent mille milliards de poèmes de Queneau sans avoir recours à la combinatoire proposée, on peut lire La vie mode d'emploi sans savoir que les appartements sont reliés les uns aux autres par la polygraphie du cavalier (et sans connaître les autres contraintes du cahier des charges). On peut lire, aussi, "El Aleph" sans réfléchir à la théorie de la fiction ni connaître les éléments mathématiques de ces nouvelles. Cependant, la connaissance de ces contraintes, de ces énigmes, de ces jeux, de la mathématique et de la logique, permet des lectures différentes, et augmente ainsi la potentialité de la lecture. Pour moi, c'est dans cette plus grande richesse de la réception que tient le principal intérêt, par rapport au lecteur, de la présence de ces aspects "mathématiques" chez Borges, Perec et les auteurs de l'Oulipo. 
D’un point de vue méthodologique, se pose alors la question de la pertinence, et des limitations, des outils théoriques de la littérature comparée pour traiter de ces questions. L'approche comparatiste traditionnelle permet évidemment de mettre en relation les œuvres de Borges et de Perec, mais les relations entre mathématique et littérature ne se placent pas sur le même plan. Ni Borges ni Perec ne sont de profonds connaisseurs de la mathématique, bien qu'ils en fassent usage, et leurs lecteurs peuvent, de même, être tout à fait ignorants des outils logiques et mathématiques, sans que cela leur interdise la lecture de ces œuvres. Il ne s'agit pas ici de lire des textes mathématiques - dans ce cas, la connaissance des règles, des théorèmes et du langage lui-même serait indispensable - mais littéraires, et la compréhension de leurs soubassements mathématiques et logiques peut alors rester tout à fait superficielle. Mais, si le lecteur connaît un peu de mathématique, il va mieux recevoir et comprendre les œuvres qui les utilisent.

Cet article avait l'intention de mettre en évidence les différences et les similitudes entre les mathématiques utilisées par Perec et par Borges, d'établir une relation entre Borges et l'Oulipo, et de montrer que l'utilisation de la mathématique et des connaissances mathématiques pouvait augmenter la richesse des textes. En effet, un lecteur qui connaît un peu de mathématique est à même de mieux comprendre et interpréter certains écrits de Perec et de Borges. On s'est donc intéressé au point de vue de la réception aussi bien qu'à celui de la production. En ce sens, Borges peut être qualifié d'oulipien puisque l'ouverture de ses écrits (quant à la production comme à la réception) en fait un écrivain potentiel.

Malgré les différences, on a pu montrer que ces deux écrivains avaient beaucoup en commun: certaines nouvelles et structures logiques, l'utilisation des éléments mathématiques comme structures et outils fictionnels. On a donc montré que la mathématique et les paradoxes sont présents chez Borges et Perec, bien que leur utilisation en soit différente: Perec a mis en œuvre ces concepts dans la structure de ses travaux, tandis que Borges y a eu recours, plus simplement, comme matériau fictionnel ${ }^{6}$.

6. Remerciements à mes directrices de thèse Madame le Professeur Christelle Reggiani (Université de Lille 3, France), Madame le Professeur Maria Esther Maciel Borges (Universidade Federal de Minas Gerais, Brésil). À Madame le Professeur Michèle Audin (Université de Strasbourg, France) et au CNPq (Conselho Nacional de Desenvolvimento Científico e Tecnológico). 


\section{Fux - 275 \\ REFERÊNCIAS BIBLIOGRÁFICAS}

BELLOS, David. Georges Perec. Une vie dans les mots. Paris: Seuil, 1994.

BORGES, Jorge Luis. Discusión. Buenos Aires: Emecé, 1957. . Atlas. Buenos Aires: Editorial Sudamérica, 1985a. . Biblioteca personal (Prólogos). Buenos Aires: Alianza, 1985b. . Sur, 1931 - 1980. Buenos Aires: Emecé, 1999. .Obras Completas. Volume 1. Barcelona: Emecé, 2002a. . Obras Completas. Volume 2. Barcelona: Emecé, 2002b. . Obras Completas. Volume 3. Barcelona: Emecé, 2002c.

CAMURATI, Mireya. Los “raros” de Borges. Buenos Aires: Corregidor, 2005.

HAYLES, N. Katherine. The cosmic web. Scientific field models E literary strategies in the $20^{\text {th }}$ century: Cornell University Press, 1984.

KASNER, Edward; Newman, James. Mathematics and the imagination. New York: Simon and Schuster, 1961.

GOLDBLOOM, William Bloch. The unimaginable mathematics of Borges' Library of Babel. New York: Oxford University Press, 2008.

LE LIONNAIS, François. Les grands courants de la pensée mathématique. Paris: Blanchard, 1948.

MAGNÉ, Bernard. Georges Perec. Paris: Nathan Université, 1999.

. "Georges Perec et les mathématiques". Revue Tangente. Paris: Editions Pole, Juillet-Août 2001. . Romans et récits. Paris: La Pochothèque, 2002.

MARTÍNEZ, Guilhermo. Borges y la matemática. Buenos Aires: Seix Barral Los Tres Mundos Ensayo, 2007.

MATHEWS, Harry. OULIPO Compendium. Atlas Press: London, 1998.

MERRELL, Floyd. Unthinking thinking: Jorge Luis Borges, mathematics, and the new physics. EUA: Purdue University Press, 1991.

OULIPO. La littérature potentielle. Paris: Gallimard, 1973.

OULIPO. Atlas de littérature potentielle. Paris: Gallimard, 1981.

PEREC, Georges. Les lettres françaises, n.1108, 1965. . Espèces d'espaces. Paris: Galilée, 1974. . W ou le souvenir d'enfance. Paris: Gallimard, 1975. . Je me souviens. Paris: Hachette, 1978a. . La vie mode d'emploi. Romans. Paris: Hachette, $1978 \mathrm{~b}$. . Récits d'Ellis Island, Paris: Le Sorbier, 1980. 
Théâtre. Paris: Hachette, 1981.

Tentative d'épuisement d'un lieu parisien. Paris: Christian Bourgois, 1982.

. Cantatrix Sopranica L. et autres écrits scientifiques. Paris: Éditions du Seuil, 1991.

. Romans et récits. Paris: La Pochothèque, 2002.

POUILLOUX, Jean-Yves. Borges fictions. Paris: Gallimard, 1992.

QUENEAU, Raymond. "La place des mathématiques dans la classification des sciences", dans François Le Lionnais,Les grands courants de la pensée mathématique. Paris: Hermann, 1998.

REGGIANI, Christelle. La rhétorique de l'invention de Raymond Roussel à l'Oulipo. Lille: A.n.r.t 1998.

ROSSET, Joseph Vidal. Qu'est-ce qu'un paradoxe? Paris: Vrin, 2004.

RUSSELL, Bertrand. Mathematical logic as based on the theory of types, American journal of mathematics 30, repris dans A source Book in Mathematical Logic 1879-1931, pp 150-182.

TURING, Alan. On computable numbers, with an Application to the Entscheidungsproblem, vol.2:42, coll. "Proceedings of the London Mathematical Societ", 1936. 